\title{
Diffracted x-ray transition radiation by a "half-bare" electron
}

\author{
S. V. Trofymenko* and N. F. Shul'ga \\ National Science Center 'Kharkiv Institute of Physics and Technology', \\ 1 Akademichna street, 61108 Kharkiv, Ukraine \\ and Karazin Kharkiv National University, 4 Svobody square, 61022 Kharkiv, Ukraine \\ A. V. Shchagin \\ National Science Center 'Kharkiv Institute of Physics and Technology', \\ 1 Akademichna street, 61108 Kharkiv, Ukraine \\ and Belgorod National Research University, 85 Pobedy street, 308015 Belgorod, Russia
}

(Received 7 December 2018; published 8 February 2019)

\begin{abstract}
The process of diffracted x-ray transition radiation by a high-energy "half-bare" electron in a crystalline target is considered in Bragg geometry. The electron is supposed to appear in the mentioned state, in which the field around it is significantly suppressed comparing to the equilibrium Coulomb one, in the result of preliminary penetration through an upstream amorphous target situated on some distance from the crystal. It is shown that the process of the electron's field regeneration after its exit from the upstream target dramatically modifies both the diffracted transition radiation angular distribution and its total yield making them dependent on the distance between the targets. Analytical expressions for these quantities, as well as their simple approximations, are derived. Almost arbitrary crystal orientation is considered. Special attention is drawn to the case of backward radiation geometry when the emitted radiation has to penetrate through the upstream target on its way to the detector.
\end{abstract}

DOI: 10.1103/PhysRevAccelBeams.22.024501

\section{INTRODUCTION}

A charged particle which enters a solid from vacuum (or gas) produces transition radiation. Its spectrum extends to frequencies of the order of $\gamma \omega_{p}$ (where $\gamma$ is the particle's Lorenz-factor and $\omega_{p}$ is the solid plasma frequency) and for high-energy particles reaches the $\mathrm{x}$-ray band. In a crystalline solid a small part of these waves experiences diffraction on crystallographic planes forming a radiation burst (which is usually called reflex) in the vicinity of the Bragg direction (the one specular to the particle velocity with respect to the planes). Such diffracted transition radiation (DTR) has been deeply studied theoretically (see, e.g., [1-4]) and observed experimentally [5]. It is shown that DTR is highly monochromatic, having frequency, which naturally equals the Bragg one, depending on the observation angle and the parameters of the crystalline lattice. It has a narrow angular distribution around the Bragg direction with the maximum intensity emitted at the angles $\vartheta \sim 1 / \gamma$ with respect to this direction.

\footnotetext{
*trofymenko@kipt.kharkov.ua
}

Published by the American Physical Society under the terms of the Creative Commons Attribution 4.0 International license. Further distribution of this work must maintain attribution to the author(s) and the published article's title, journal citation, and DOI.
Transition radiation is not a single field which undergoes diffraction in the considered case. Another part of the emission in the Bragg direction, known as parametric x-ray radiation (PXR) [2-4,6-11], comes from the diffraction of virtual photons constituting the particle's proper field inside the crystal. At high particle energies its distribution has the angular width $\sim \omega_{p} / \omega$ (where $\omega$ is the radiation frequency) which significantly exceeds the one $\sim 1 / \gamma$ of DTR. These types of emission also have slightly different frequencies and the interference between them is usually very weak. The mentioned facts provide a principle possibility to consider these emissions separately from each other. At $\gamma \gg$ $\omega / \omega_{p}$ the PXR intensity saturates while the one of DTR continues growing with the increase of $\gamma$. This makes DTR dominating at high particle energies, at least at the angles $\vartheta \ll \omega_{p} / \omega$, and it is this type of emission which we will focus upon in the present paper.

In this work we study the properties of DTR in a special case, when they considerably differ from the usual properties of this radiation described above. In this case DTR is produced by a so-called "half-bare" electron (the term was firstly introduced in [12]) impinging upon a crystal. Such electron does not have an ordinary Coulomb field around itself, which, as we show, results in a modified angular distribution and yield of DTR. The electron can appear in the "half-bare" state in the result of interaction (before impinging upon the crystal) with substance or external 
fields. For instance, the electron traversal of an exit window of a vacuum chamber or any other obstacles on the beam line (scintillators, telescopes etc.) can serve as examples of such interaction and may be unavoidable in the experiment. The particle preserves such a state within the distance from the interaction region known as formation length $l_{F}$. It can be roughly estimated as $l_{F} \sim \gamma^{2} / \omega$. At sufficiently high particle energies $l_{F}$ can be macroscopically large even in the X-ray band of frequencies. In the present paper we investigate the modification of DTR characteristics in the case when the crystal is situated within the formation distance from the region where the electron "undresses."

The effects associated with the discussed state of a particle and large size of the formation distances were first studied theoretically [13-16] and observed experimentally $[17,18]$ for the electron bremsstrahlung in amorphous media. In recent years the analogous effects were confirmed to exist for the millimeter wavelength diffraction radiation [19]. In $[20,21]$ the modification of the coherent $\mathrm{x}$-ray emission by a high-energy electron in an ultrathin crystal in the result of the particle preliminary penetration through a thick amorphous target was investigated. Here the discussed emission consisted of a strongly interfering (due to small crystal thickness) sum of DTR and PXR which could not be separated from each other. In the present work we consider a case which seems to be more appropriate from the experimental point of view. We still apply an upstream target to "undress" the particle, but the studied emission is DTR in Bragg geometry from a crystal, which can be arbitrarily thick. Such emission is formed in a thin boundary layer of the crystal known as extinction length. Thus its characteristics are sensitive to the state of the impinging electron's field, just like in the mentioned case of an ultrathin crystal (it is not the case for PXR formed in a much thicker layer of the crystal). Almost arbitrary crystal orientation is considered here. Special attention is drawn to the backward radiation geometry.

The process of DTR from several successively located targets has been considered either theoretically or experimentally in a series of papers (see, e.g., [22-24]). The main attention in these works is rather paid to the purpose of an intense X-ray sources development on the basis of the combined DTR and PXR emission from multilayer structures. For instance, in [24] as a particular case the authors discuss a two-target geometry (analogous to the one considered in the present paper). The major attention there is drawn to the study of radiation angular distribution under conditions of the most constructive interference of emissions from the both targets. In our work it is the evolution of DTR properties (such as its angular distribution and integrated radiation yield) with the change of the distance between the targets within the formation length which we focus on. Such evolution is studied as a manifestation of the process of the electron's field regeneration after the particle penetration through the amorphous target. The optimal conditions for the experimental observation of the considered effect are discussed.

Let us also note that a comprehensive study of DTR is not only of fundamental interest but of practical one as well. For instance, in [25,26] it was proposed to use such emission for diagnostics of high-energy charged particle beams. This is expected to bring new possibilities for diagnostics of beams of micrometer-scale (and smaller) transverse size together with the analogous techniques based on PXR [27,28] and optical transition radiation (see, e.g., $[29,30]$ ).

\section{ELECTRON'S FIELD EVOLUTION}

Let us consider a process in which a high-energy electron, moving along the $z$ axis with the velocity $\mathrm{v}$ penetrates through an upstream target and impinges upon a downstream crystal producing here a DTR reflex (Fig. 1). We will consider this process in the Bragg geometry assuming that the chosen set of crystallographic planes is parallel to the crystal surface. The crystalline target may have an arbitrary inclination with respect to the $z$ axis, which defines the Bragg angle $\theta_{B}$ in the present case. The upstream target is regarded here as amorphous in the sense that its crystalline structure (in case it exists) does not manifest itself in the properties of radiation from the downstream crystal. Both targets are assumed to be installed in vacuum. The properties of the discussed reflex are defined by the field around the electron in vacuum which falls on the crystal generating the transition radiation field, which undergoes diffraction. Therefore, let us first discuss some aspects of evolution of the field in the region between the targets.

For simplicity in Secs. II and III we assume that the upstream target is thick enough to absorb the transition radiation which is emitted on its upstream surface. In this

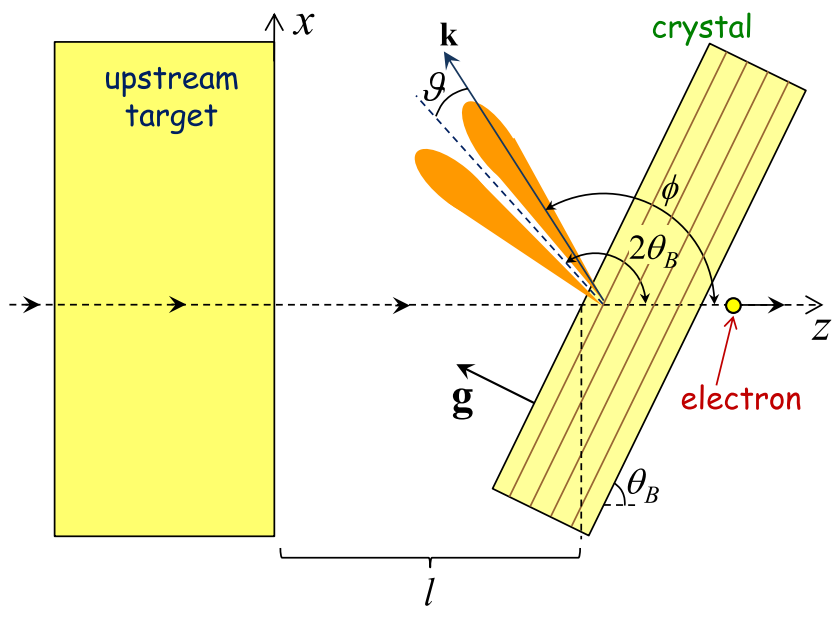

FIG. 1. Electron penetration through the upstream target and its further incidence upon the crystal, accompanied by DTR emission. 
case it is only the boundary condition on its downstream surface that defines the field around the electron in vacuum. In ultrarelativistic case this field can be approximately considered as perpendicular to the $z$ axis. At frequencies under consideration, which are higher than the characteristic atomic ones, we can use an asymptotic form of the dielectric permittivity of the upstream target: $\varepsilon(\omega)=1-\eta_{p}^{2} / \omega^{2}$, where $\eta_{p}$ is the target plasma frequency. By $\omega_{p}$ we will denote such frequency of the crystal. In this case the field between the targets reads $[6,20]$ :

$$
\begin{aligned}
\mathbf{E}(\omega, \mathbf{r})= & -\frac{i e}{\pi} \int d^{2} \mathrm{q} \mathbf{q}\left\{F(\mathrm{q}) e^{i \omega z / \mathrm{v}}\right. \\
& \left.+G\left(\mathrm{q}, \eta_{p}\right) e^{i z \sqrt{\omega^{2}-\mathrm{q}^{2}}}\right\} e^{i \mathbf{q} \boldsymbol{\rho}},
\end{aligned}
$$

in which $F(\mathrm{q})=1 /\left(\mathrm{q}^{2}+\omega^{2} / \gamma^{2}\right)$ and $G\left(\mathrm{q}, \eta_{p}\right)=1 /\left(\mathrm{q}^{2}+\right.$ $\left.\eta_{p}^{2}+\omega^{2} / \gamma^{2}\right)-F(\mathrm{q})$ and the beginning of the $z$ axis is chosen at the downstream surface of the amorphous target. Here $\rho$ is the radius-vector of the observation point in the $x y$ plane, while $\mathbf{q}$ is the wave-vector component in this plane. We use a system of units in which the speed of light and the Planck constant equal unity. However, for convenience, these quantities will be recovered in the expressions describing the main results of the paper.

At $z \rightarrow 0$, just after the electron exits from the upstream target, the field (1) is simplified to

$$
\mathbf{E}(\omega, \mathbf{r}) \approx 2 e \eta_{p} \frac{\boldsymbol{\rho}}{\rho} e^{i \omega z} K_{1}\left(\eta_{p} \rho\right),
$$

and is still equal to the Fourier component of the electron's proper field inside the target. Here $K_{1}(x)$ is the Macdonald function. The expression (2) indicates that in the considered region the Fourier components of the field around the electron with frequencies $\omega<\gamma \eta_{p}$ are suppressed comparing to such components of the electron's proper field in vacuum (which is defined by (2) with a substitution $\left.\eta_{p} \rightarrow \omega / \gamma\right)$. This makes such electron "half-bare." With the increase of $z$ the regeneration of these low-frequency components takes place. This process can be most directly illustrated if consider the electromagnetic energy flux associated with the field (1) which crosses a plane $z=$ const.

In the considered ultrarelativistic limit the magnetic field around the electron is just $\mathbf{H}(\omega, \mathbf{r}) \approx \mathbf{e}_{z} \times \mathbf{E}(\omega, \mathbf{r})$. It allows us to write the amount of energy which traverses a unitary surface during the whole observation time interval in a standard form, like in the case of an electromagnetic wave:

$$
\frac{d J(\omega, \mathbf{r})}{d \omega d^{2} \rho}=|\mathbf{E}(\omega, \mathbf{r})|^{2} / 4 \pi^{2} .
$$

The integration of this quantity over the entire plane $z=$ const with the use of (1) gives the total amount of energy in the unitary range of frequencies which traverses this plane. According to (2), the field around the electron inside the target (and immediately after the particle exit from it) is different from the Coulomb field in vacuum on distances $\rho>1 / \eta_{p}$ from the electron trajectory, while it remains intact by the substance polarization at smaller $\rho$. Therefore in order to estimate just the varying (with the increase of $z$ ) part of the discussed energy flux it is enough to integrate (3) applying as a lower limit $\rho \sim 1 / \eta_{p}$. Then in the region of distances $\omega / \eta_{p}^{2} \ll z \ll \gamma^{2} / \omega$ with the logarithmic accuracy we obtain:

$$
\frac{d J(\omega, z)}{d \omega}=\frac{2 e^{2}}{\pi} \ln \frac{\eta_{p}^{2} z}{\omega} .
$$

Here we see that within the formation length $l_{F} \sim \gamma^{2} / \omega$ there occurs a logarithmic growth of the electromagnetic energy flux around the electron with the increase of $z$. The energy balance is provided by the decrease of the electron kinetic energy in this case (which we, naturally, neglect). Such change of the energy flux leads to variation of the DTR yield with the change of the crystal position within $z \sim l_{F}$. Moreover, the discussed field evolution is accompanied by the redistribution of the energy of the field (1) with respect to $\mathbf{q}$, which leads to variation of the DTR angular distribution. The mentioned effects are studied further in detail.

\section{DTR FROM AN INCLINED TARGET}

Let us consider the properties of DTR in the geometry depicted on Fig. 1, when the crystalline target has some inclination $\theta_{B}$ to the $z$ axis. For this aim it is necessary to calculate the transition radiation field inside the crystal, which undergoes diffraction. This can be done with the use of the expression (1) for the impinging field and the boundary conditions at the crystal surface. These conditions imply a continuity of the electric field tangential components and the normal ones of the induction. Accounting for the proximity of the crystal dielectric permittivity to unity in the considered frequency range ( $\omega$ is about several $\mathrm{keV}$ ) the latter condition can be approximately rewritten to state the continuity of the electric field normal components. This results in the approximate continuity of the total electric field vector on the crystal surface.

The field inside the crystal consists of the transition radiation field and the electron's proper field. The first one can be presented in a standard form [6] as

$$
\mathbf{E}_{t r}(\omega, \mathbf{r})=\int d^{2} \mathrm{q} \mathbf{E}_{\mathrm{tr}}(\omega, \mathbf{q}) e^{i z \sqrt{\omega^{2} \varepsilon-\mathrm{q}^{2}}+i \mathbf{q} \rho},
$$

while the second one is defined by the first term in (1) with the substitution $F(\mathrm{q}) \rightarrow F\left(\mathrm{q}, \omega_{p}\right)=1 /\left(\mathrm{q}^{2}+\omega_{p}^{2}+\omega^{2} / \gamma^{2}\right)$. The crystal boundary equation can be written as $z=z(\boldsymbol{\rho})=$ $l+\boldsymbol{\rho} \mathbf{e}_{x} \operatorname{ctg} \theta_{B}$, where $\mathbf{e}_{x}$ is a unit vector in the direction of the $x$ axis. The boundary condition gives the following expression for the transition radiation field in the immediate vicinity of the crystal surface for a certain value of $\rho$ : 


$$
\mathbf{E}_{\mathrm{tr}}(\omega, \boldsymbol{\rho})=\frac{i e}{\pi} \int d^{2} \mathbf{q} \mathbf{q} f(\mathbf{q}, \boldsymbol{\rho}) e^{i \mathbf{q} \rho}
$$

with

$$
f(\mathrm{q}, \boldsymbol{\rho})=G\left(\mathrm{q}, \omega_{p}\right) e^{i \omega z(\boldsymbol{\rho}) / \mathrm{v}}-G\left(\mathrm{q}, \eta_{p}\right) e^{i z(\boldsymbol{\rho}) \sqrt{\omega^{2}-\mathrm{q}^{2}}} .
$$

With the use of (3) and (6) the total spectral density of the electromagnetic energy associated with the transition radiation can be calculated as

$$
\frac{d J_{\mathrm{tr}}(\omega)}{d \omega}=\int d^{2} \rho d^{2} \mathbf{q} d^{2} \mathrm{q}^{\prime} \mathbf{q} \mathbf{q}^{\prime} f(\mathrm{q}, \boldsymbol{\rho}) f^{*}\left(\mathrm{q}^{\prime}, \boldsymbol{\rho}\right) / 4 \pi^{2} .
$$

The terms in the integrand in (7) containing $G\left(\mathrm{q}, \omega_{p}\right)$ $G\left(\mathrm{q}^{\prime}, \eta_{p}\right)$ and the complex conjugate of it possess exponents of the type $e^{i \mathbf{\rho} \mathbf{e}_{x} \operatorname{ctg} \theta_{B}\left(\omega / \mathrm{v}-\sqrt{\omega^{2}-\mathrm{q}^{2}}\right)}$. In ultrarelativistic case the condition $\mathrm{q} \ll \omega$ is valid for the values of $\mathrm{q}$ making the main contribution to (6). It illustrates the fact that the transition radiation emission happens predominantly at small angles with respect to the particle velocity (namely, $\mathrm{q} / \omega \sim 1 / \gamma)$. Thus the discussed exponent can be written as $e^{i \boldsymbol{\rho} \mathbf{e}_{x} \operatorname{ctg} \theta_{B}\left(\gamma^{-2}+\mathrm{q}^{2} / \omega^{2}\right) \omega / 2}$. The characteristic transversal (with respect to the $z$ axis) size of the electron's Coulomb field in vacuum is of the order of $\gamma / \omega$. This makes the effective values of the expression in the discussed exponent not exceed $\operatorname{ctg} \theta_{B} / \gamma$. Therefore, if the crystalline target inclination satisfies the condition $\theta_{B} \gg 1 / \gamma$, which we will further assume to be valid, the discussed exponent can be set to equal unity. In this case the integrals in (7) with respect to $\rho$ and $\mathrm{q}^{\prime}$ can be simply calculated which gives

$$
\begin{aligned}
\frac{d J_{\mathrm{tr}}(\omega)}{d \omega}= & \frac{e^{2}}{\pi^{2}} \int d^{2} \mathrm{qq}^{2}\left\{G^{2}\left(\mathrm{q}, \omega_{p}\right)+G^{2}\left(\mathrm{q}, \eta_{p}\right)\right. \\
& -2 G\left(\mathrm{q}, \omega_{p}\right) G\left(\mathrm{q}, \eta_{p}\right) \\
& \left.\times \cos \left[\omega l\left(\gamma^{-2}+\mathrm{q}^{2} / \omega^{2}\right) / 2\right]\right\}
\end{aligned}
$$

Presenting transversal component $\mathrm{q}$ of the transition radiation wave vector as $\mathrm{q}=\omega \beta$ (here again we apply $\varepsilon_{c r} \approx 1$ for the dielectric permittivity of the crystal) from (8) the angular distribution of the radiation energy is directly obtained:

$$
\begin{aligned}
\frac{d^{2} J_{\mathrm{tr}}(\omega)}{d \omega d o}= & \frac{e^{2} \beta^{2}}{\pi^{2}}\left\{G_{1}^{2}\left(\beta, \omega_{p}\right)+G_{1}^{2}\left(\beta, \eta_{p}\right)\right. \\
& -2 G_{1}\left(\beta, \omega_{p}\right) G_{1}\left(\beta, \eta_{p}\right) \\
& \left.\times \cos \left[\omega l\left(\gamma^{-2}+\beta^{2}\right) / 2\right]\right\},
\end{aligned}
$$

where $G_{1}(\beta, x)=\left(\beta^{2}+\gamma^{-2}+x^{2} / \omega^{2}\right)^{-1}-\left(\beta^{2}+\gamma^{-2}\right)^{-1}$.

The resulting DTR angular distribution can be obtained multiplying (9) by the crystal reflection coefficient $R(\omega)$ and integrating the obtained expression with respect to $\omega$. Such procedure can be easily done with the use of the following relation [31], valid in the case when the considered crystallographic planes are parallel to the crystal surface:

$$
\int R(\omega) d \omega / \omega=\frac{16}{3}\left(\frac{\omega_{B}}{c \mathrm{~g}}\right)^{2}\left|\chi_{\mathbf{g}}\right| P,
$$

in which, for convenience, we preserved the speed of light $c$. Here $\mathrm{g}$ is the absolute value of the reciprocal lattice vector $\mathbf{g}$ corresponding to the chosen set of planes, $\chi_{\mathbf{g}}$ are the coefficients in the Fourier series decomposition of the crystal dielectric susceptibility $\chi=1-\varepsilon_{c r}$ with respect to g, $P$ is the polarization factor which equals unity for perpendicular polarization and $|\cos \phi|$ for the parallel one. The type of polarization is defined here with respect to the plane of vector $\mathbf{g}$ and the particle velocity vector $\mathbf{v}$, while $\phi$ is the angle between the DTR wave vector $\mathbf{k}$ and the $z$ axis (see Fig. 1).

The reflection coefficient $R(\omega)$ is different from zero, being very close to unity, within a very narrow frequency region (known as Darwin table) around the Bragg frequency $\omega_{B}$. Such frequency is defined by the formula $\omega_{B}=$ $c \mathrm{~g} /[2 \sin (\phi / 2)]$ and determines the DTR frequency in the direction of observation. In the considered case of sufficiently high electron energies we will neglect $\omega_{B}$ variation within the major part of the reflex and put here $\phi=2 \theta_{B}$. A small width of the Darwin table (which is of the order of $1 \mathrm{eV}$ in the present case) allows also neglecting the frequency variation in the integrand in (10) within the effective integration region. In the result, with the use of (9) and (10), the angular distribution of the number of DTR photons can be obtained in the following form:

$$
\begin{aligned}
\frac{d N}{d \Omega}= & \frac{16 \alpha\left|\chi_{\mathbf{g}}\right|}{3 \pi^{2}}\left(\frac{\omega_{B}}{c \mathrm{~g}}\right)^{2}\left(\vartheta_{\perp}^{2}+|\cos \phi| \vartheta_{\|}^{2}\right)\left\{G_{1}^{2}\left(\vartheta, \omega_{p}\right)\right. \\
& \left.+G_{1}^{2}\left(\vartheta, \eta_{p}\right)-2 G_{1}\left(\vartheta, \omega_{p}\right) G_{1}\left(\vartheta, \eta_{p}\right) \cos \left(l / l_{F}\right)\right\},
\end{aligned}
$$

where $l_{F}=2 \omega^{-1} /\left(\gamma^{-2}+\vartheta^{2}\right)$ is the formation length and $\alpha$ is the fine-structure constant. Here also $\vartheta_{\|}, \vartheta_{\perp} \ll 1$ are the components of the emission angle $\boldsymbol{\vartheta}$ (counted from the Bragg direction $\phi=2 \theta_{B}$ ) parallel and perpendicular to the plane of the vectors $\mathbf{v}$ and $\mathbf{g}$.

Further we will make estimations for (220) plane of a silicon crystal $\left(\omega_{p} \approx 31 \mathrm{eV}\right)$ for which the numerical value of (10) for $P=1$ is $7.45 \times 10^{-5}$. It does not depend on the crystal orientation and $\omega_{B}$ (the $\omega_{B}$ dependence of the quantity $\chi_{\mathrm{g}}$ contracts with the one in the prefactor).

Figure 2 shows the examples of the DTR angular distribution for the case of pure perpendicular polarization $\left(\vartheta_{\|}=0\right)$ for different values of the particle path length $l$ between the targets (solid lines). The electron energy is taken to equal $10 \mathrm{GeV}$. In the presented angular range $\left(\omega_{B} \vartheta_{\perp} \ll\right.$ $\left.\omega_{p}, \eta_{p}\right)$ these distributions do not depend on the angle of the crystalline target inclination. For comparison, the dotted 


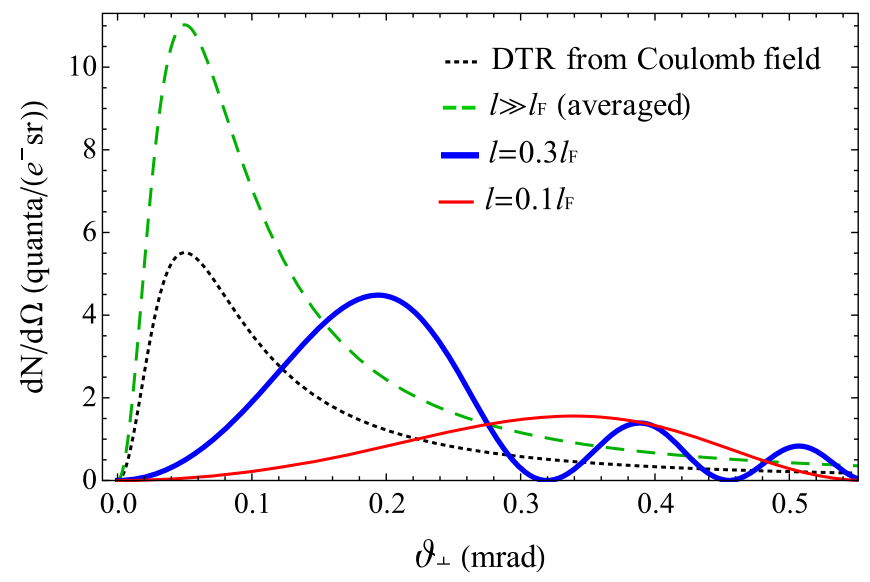

FIG. 2. Angular distribution of the number of DTR photons for different distances $l$ between the targets for $\vartheta_{\|}=0$. The electron energy is $10 \mathrm{GeV}$.

line shows the corresponding DTR distribution $[4,26]$ in the case of the impinging electron with Coulomb field (in the absence of the upstream target). Here we see that at $l<l_{F}\left(l_{F}\right.$ is defined for $\vartheta=1 / \gamma$ ) the DTR angular distribution is much wider than the customary one with the maximum position at $\vartheta=1 / \gamma$ (in case $\gamma \gg \omega / \omega_{p}$ ). With the increase of $l$ the main maximum position shifts to the smaller values of $\vartheta_{\perp}$ and the additional maxima grow in their amplitude. At $l \gg l_{F}$ the distribution turns into quick oscillations. In this case it is practical to average it with respect to small angular intervals. Such asymptotic average behavior of the number of radiated quanta is presented by the dashed line.

As the figure shows, at $l \gg l_{F}$ the radiation intensity still exceeds the one typical for the electron with the Coulomb field (dotted line). This happens due to the additional contribution of the transition radiation from the upstream target which experiences Bragg diffraction in the crystal. At $z<l_{F}$ the forming field of this radiation cannot be separated from the electron's proper Coulomb field. The destructive interference of these fields leads to the suppression of the electron's proper field and causes the effect of "half-bareness." At $z>l_{F}$ the mentioned interference disappears and we deal with an incoherent sum of the contributions from the electron's proper field and the field of the discussed transition radiation.

In the intermediate range of distances $\omega / \eta_{p}^{2} \ll z \ll \gamma^{2} / \omega$, corresponding to the applicability region of (4), the expression (11) for the DTR angular distribution can be simplified. In this case characteristic values of the emission angles are $\vartheta \gg 1 / \gamma$. At the considered high electron energies $(\gamma \gg$ $\omega / \omega_{p}$ ) such angles are still much less than $\omega_{p} / \omega$ (as well as $\left.\eta_{p} / \omega\right)$. This gives $G_{1}\left(\vartheta, \omega_{p}\right) \approx G_{1}\left(\vartheta, \eta_{p}\right) \approx \vartheta^{-2}$ and (11) transforms into

$\frac{d N}{d \Omega}=\frac{16 \alpha\left|\chi_{\mathbf{g}}\right|}{3 \pi^{2}}\left(\frac{\omega_{B}}{c \mathrm{~g}}\right)^{2} \frac{4\left(\vartheta_{\perp}^{2}+|\cos \phi| \vartheta_{\|}^{2}\right)}{\vartheta^{4}} \sin ^{2}\left(\frac{l \vartheta^{2}}{4 \omega_{B}}\right)$.

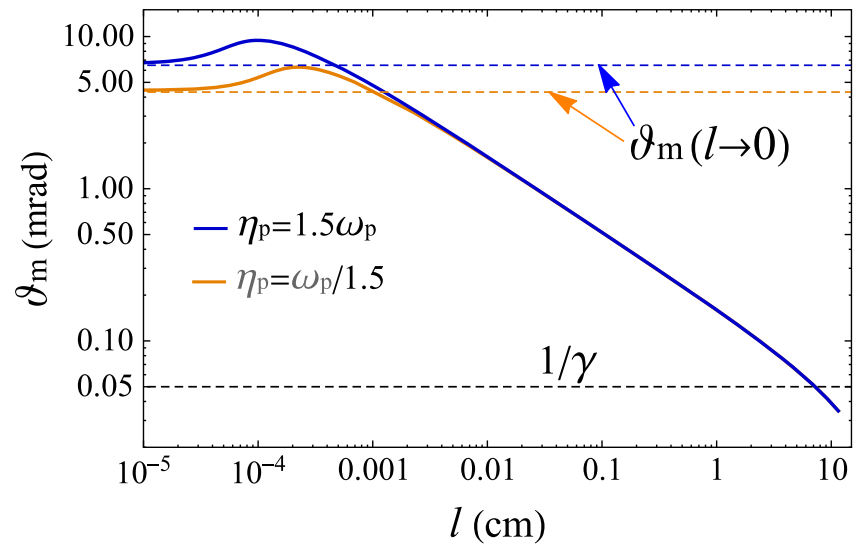

FIG. 3. Evolution of the maximum position of DTR angular distribution with the increase of $l$ for two values of the upstream target plasma frequency $\eta_{p}$. The electron energy is $10 \mathrm{GeV}$.

On the basis of this expression the formula for the main maximum position of the radiation angular distribution in the considered region of $l$ can be derived. For simplicity let us consider the radiation with a pure perpendicular polarization $\left(\vartheta_{\|}=0\right)$. In this case the main maximum position $\vartheta_{\perp}=\vartheta_{m}$ of (12) is defined by a transcendental equation $\operatorname{tg} x=2 x$ with $x=l \vartheta_{m}^{2} /\left(4 \omega_{B}\right)$. Its numerical solution gives

$$
\vartheta_{m}(l) \approx \sqrt{\frac{4.66}{\omega_{B} l}} .
$$

The same result is naturally obtained in the case of a pure parallel radiation polarization $\left(\vartheta_{\perp}=0\right)$. It is also valid in the case $|\cos \phi| \approx 1$, which takes place if $\theta_{B}$ is not far either from $\pi / 2$ or from zero, provided the condition $\theta_{B} \gg 1 / \gamma$ is fulfilled. In this case $\vartheta_{m}$ is the value of $\vartheta$ corresponding to the maximum of an almost azimuthally symmetric DTR distribution.

Figure 3 shows the dependence of $\vartheta_{m}$ on $l$. It depicts the result of numerical calculation for the case $\vartheta_{\|}=0$ (as in Fig. 2) and $\theta_{B}=75^{\circ}$ (which corresponds to $\omega_{B} \approx 3.343 \mathrm{keV}$ ) on the basis of (11) for arbitrary $l$, which nicely coincides with (13) in the applicability region of this formula. For the chosen electron energy $(10 \mathrm{GeV})$ the formation length for $\vartheta=0$ is presently $l_{F} \approx 4.5 \mathrm{~cm}$. The figure shows that at high electron energies with the change of the distance between the targets the value of $\vartheta_{m}$ experiences a variation by several orders of magnitude. At very small values of $l$ this variation is not monotonic. At $l \gg l_{F}$ the value of $\vartheta_{m}$ ceases to characterize the angular distribution since, as mentioned previously, $d N / d \Omega$ in this case has a lot of maxima and turns into quick oscillations. However, after the averaging over such oscillations the maximum position naturally settles at $1 / \gamma$, as Fig. 2 shows.

The limit value of $\vartheta_{m}$ at $l \rightarrow 0$ is uniquely defined by $\omega_{p}$, $\eta_{p}$ and $\omega_{B}$ and can be easily obtained from (11). We do not present here the corresponding formula. It is worth noting 


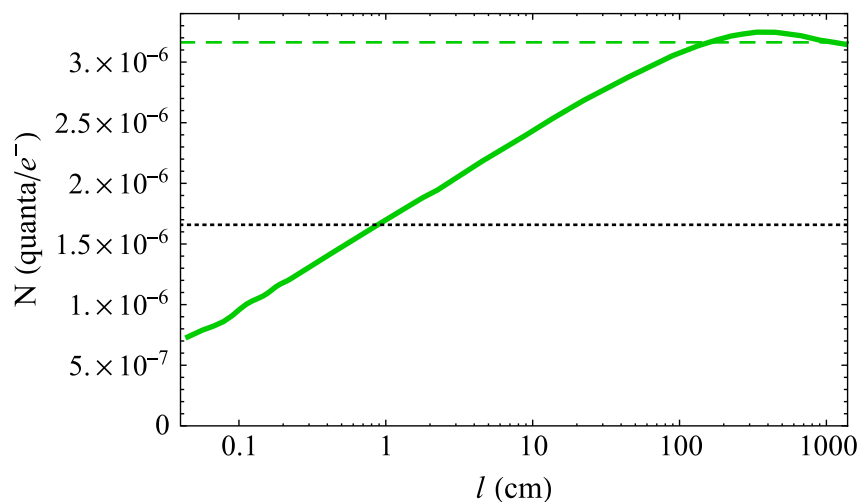

FIG. 4. Dependence of the angle-integrated DTR yield on $l$ for the electron energy $100 \mathrm{GeV}$ and the acceptance angle $2 \vartheta_{0}=2 \mathrm{mrad}$. Calculation on the basis of (11) for $\theta_{B}=75^{\circ}$. Dashed line-asymptote at $l \gg l_{F}$. Dotted line-DTR yield in the absence of the upstream target.

that in the considered case of an inclined crystalline target sufficiently small values of $l$ are, certainly, experimentally unachievable and present just a theoretical interest. However, the situation changes in a modified statement of this problem discussed in Sec. IV. In this section the case of a backward radiation geometry is studied in more detail. Such geometry allows, in principle, performing measurements at arbitrary values of $l$.

The evolution of the electron's field in the considered process after the particle exit from the upstream target can be visualized most explicitly if consider the dependence of the angle-integrated DTR yield on distance $l$ between the targets. Fig. 4 shows the result of the angle integration of the distribution (11) in the interval $-\vartheta_{0}<\vartheta_{\perp}, \vartheta_{\|}<\vartheta_{0}$, with $\vartheta_{0}=200 / \gamma$. The electron energy is chosen as $100 \mathrm{GeV}$, which makes the variation range of $\vartheta_{\perp}$ and $\vartheta_{\|}$ equal $2 \mathrm{mrad}$. The figure indicates a noticeable increase of the DTR yield with the increase of the distance between the targets up to $l \sim l_{F}$, while in the case under discussion $l_{F} \approx$ $4.5 \mathrm{~m}$ (for $\vartheta=0$ ).

On the basis of (12) it is possible to derive a simple analytical expression for the DTR yield in the intermediate range of distances $\omega / \eta_{p}^{2} \ll l \ll \gamma^{2} / \omega$. For the additional simplification let us assume the approximation $|\cos \phi| \approx 1$ to be valid. In this case, integrating (12) with respect to $\vartheta$ up to $\vartheta=\vartheta_{0}$, for the radiation yield we obtain:

$$
N\left(l, \vartheta_{0}\right)=\frac{32 \alpha\left|\chi_{\mathbf{g}}\right|}{3 \pi}\left(\frac{\omega_{B}}{c \mathrm{~g}}\right)^{2}\left\{\Gamma+\ln \frac{\omega_{B} l \vartheta_{0}^{2}}{2}\right\},
$$

where $\Gamma \approx 0.577$ is the Euler's constant and it is assumed that $\vartheta_{0} \gg 1 / \gamma$. This expression indicates the logarithmic character of the DTR yield increase, which reflects the analogous character of the electromagnetic energy flux variation (4) in the region between the targets.

\section{BACKWARD RADIATION GEOMETRY}

Let us consider the discussed process in the case when $\theta_{B}=\pi / 2$, or close to it, and the targets are parallel to each other. This case is of special interest since it provides a principle possibility to perform measurements at arbitrary distances $l$ between the targets. Previously, for simplicity, we considered the upstream target as rather thick and supposed that DTR did not have to penetrate through it on its way to a detector. In the present case the radiation is emitted in the backward direction with respect to the particle velocity and such penetration becomes unavoidable. Presently the upstream target has to be thin enough to let a considerable part of DTR to penetrate through it, as well as sufficiently thick to "undress" the electron. This means that the value of the target thickness $L$ should lie between the DTR absorption length $L_{\mathrm{abs}}$ and the radiation formation length inside it, which we denote as $L_{F}$.

Let us, for the sake of convenience, choose the beginning of the $z$ axis on the upstream surface of the amorphous target. In the considered case the transition radiation from this surface is not considerably absorbed in this target and the boundary condition at $z=0$ should be taken into account in the calculations of the field in this process. Taking this into account and performing a procedure analogous to the one from the previous section, for the field of transition radiation inside the crystal in the immediate vicinity of its surface $(z=L+l)$ we obtain:

$$
\begin{aligned}
\mathbf{E}_{\mathrm{tr}}(\omega, \boldsymbol{\rho})= & \frac{i e}{\pi} e^{i \omega(L+l) / \mathrm{v}} \int d^{2} \mathrm{q} \mathbf{q} e^{i \mathbf{q} \rho}\left\{G\left(\mathrm{q}, \omega_{p}\right)\right. \\
& -G\left(\mathrm{q}, \eta_{p}\right) e^{-i \omega l\left(\gamma^{-2}+q^{2} / \omega^{2}\right) / 2} \\
& \left.\times\left(1-e^{-L\left[i \omega\left(\gamma^{-2}+\left(q^{2}+\eta_{p}^{2}\right) / \omega^{2}\right)+\sigma\right] / 2}\right)\right\},
\end{aligned}
$$

where $\sigma$ is the energy absorption coefficient for the considered frequency. With the use of (15), (3), and (10) the expression for the DTR angular distribution (after its penetration through the upstream target) in the present case can be derived:

$$
\begin{aligned}
\frac{d N}{d \Omega}= & \frac{16 \alpha\left|\chi_{\mathbf{g}}\right|}{3 \pi^{2}}\left(\frac{\omega_{B}}{c \mathrm{~g}}\right)^{2} e^{-L \sigma} \vartheta^{2}\left\{G_{1}^{2}\left(\vartheta, \omega_{p}\right)\right. \\
& +G_{1}^{2}\left(\vartheta, \eta_{p}\right)\left[1+e^{-L \sigma}-2 e^{-L \sigma / 2} \cos \left(L / L_{F}\right)\right] \\
& -2 G_{1}\left(\vartheta, \omega_{p}\right) G_{1}\left(\vartheta, \eta_{p}\right) \\
& \left.\times\left[\cos \left(\frac{l}{l_{F}}\right)-e^{-L \sigma / 2} \cos \left(\frac{l}{l_{F}}+\frac{L}{L_{F}}\right)\right]\right\},
\end{aligned}
$$

where $L_{F}=2 \omega_{B}^{-1} /\left(\gamma^{-2}+\vartheta^{2}+\eta_{p}^{2} / \omega_{B}^{2}\right)$ is the formation length inside the upstream target.

The analogue of the simplified expression (14) in the present case, approximately describing the DTR yield evolution in the range of distances $\omega / \eta_{p}^{2} \ll l \ll \gamma^{2} / \omega$, reads: 


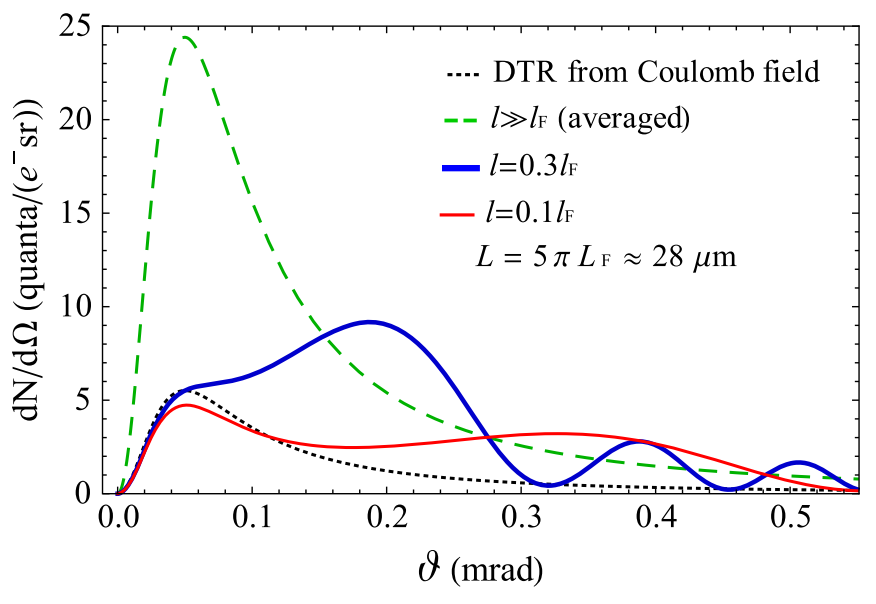

FIG. 5. Angular distribution of the number of DTR photons for the backward radiation geometry and a beryllium upstream target of $L=5 \pi L_{F} \approx 28 \mu \mathrm{m}$. The electron energy is $10 \mathrm{GeV}$. The curves have the same meaning as in Fig. 2.

$$
\begin{aligned}
N\left(l, \vartheta_{0}\right)= & \frac{32 \alpha\left|\chi_{\mathbf{g}}\right|}{3 \pi}\left(\frac{\omega_{B}}{c \mathrm{~g}}\right)^{2} e^{-L \sigma}\left\{\left[\ln \left(\gamma \vartheta_{0}\right)-1 / 2\right] e^{-L \sigma}\right. \\
& +\left[1-e^{-L \sigma / 2} \cos \left(\frac{L \eta_{p}^{2}}{2 \omega_{B}}\right)\right]\left[\Gamma+\ln \frac{\omega_{B} l \vartheta_{0}^{2}}{2}\right] \\
& \left.-\frac{\pi}{2} e^{-L \sigma / 2} \sin \left(\frac{L \eta_{p}^{2}}{2 \omega_{B}}\right)\right\} .
\end{aligned}
$$

Let us make estimations for the case of a beryllium upstream target. It is very suitable since in this case $L_{\mathrm{abs}} \approx$ $320 \mu \mathrm{m}$ and $L_{F} \approx 2 \omega_{B} / \eta_{p}^{2} \approx 1.8 \mu \mathrm{m}$ and there is a huge gap between these quantities to be occupied by the values of $L$. Here we took $\eta_{p}=26.1 \mathrm{eV}$ and $\omega_{B}=3.229 \mathrm{keV}$, which corresponds to $2 \theta_{B}=\pi$. The estimation of $L_{F}$ is made for $\vartheta \ll \eta_{p} / \omega_{B}$. The expressions (16) and (17) manifest an oscillatory dependence on the upstream target thickness. These formulas indicate that it is natural to expect the highest radiation intensity for $L \approx(2 n+1) \pi L_{F}$, where $n$ is an integer. Figure 5 shows the angular distributions of the number of DTR photons for the case $L=5 \pi L_{F} \approx 28 \mu \mathrm{m}$. For convenience the curves are presented for the same values of $l$ as in Fig. 2. Here we see that in the present case the DTR angular distribution is analogous to the one in the case of the inclined geometry. The difference is, however, significant for $\vartheta \sim 1 / \gamma$ due to the influence of the upstream surface of the beryllium target. Moreover, in the considered case the radiation intensity is noticeably higher.

The curves depicting the evolution of the DTR yield in the present case are shown in Fig. 6. Here three cases are presented, corresponding to a beryllium upstream target of thickness $L=5 \pi L_{F}, L=15 \pi L_{F}$ and $L=6 \pi L_{F}$. In the first two cases, which are the special ones of $L \approx$ $(2 n+1) \pi L_{F}$ ( $n$ is an integer) we observe a noticeable change of the DTR yield within $l \sim l_{F}$. In the third case,

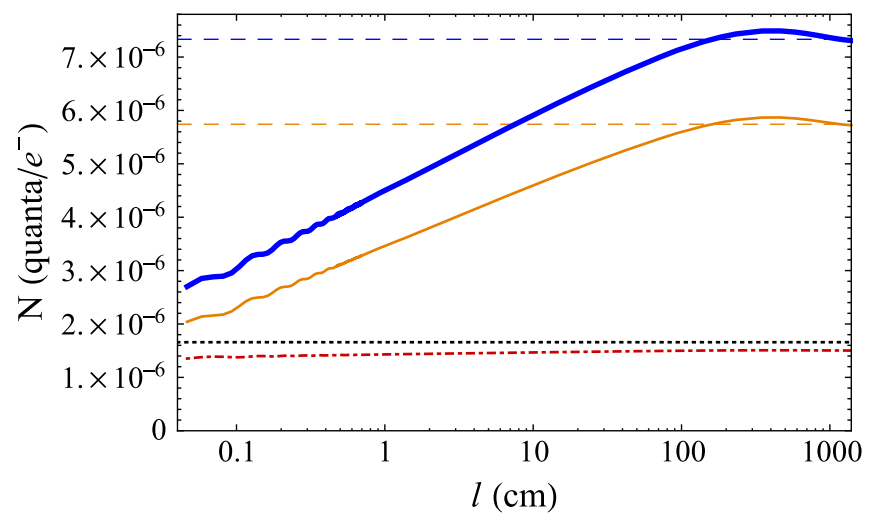

FIG. 6. Dependence of the angle-integrated DTR yield on $l$ for the electron energy $100 \mathrm{GeV}$ and the acceptance angle $2 \vartheta_{0}=$ $2 \mathrm{mrad}$ in the backward radiation geometry. Calculation on the basis of (16) for a beryllium upstream target thickness $L=$ $5 \pi L_{F} \approx 28 \mu \mathrm{m}$ (thick blue line), $L=15 \pi L_{F} \approx 84 \mu \mathrm{m}$ (thin orange line) and $L=6 \pi L_{F} \approx 33.5 \mu \mathrm{m}$ (dot-dashed line). Dotted line-the same as in Fig. 4.

like in the analogous ones in which $L \approx 2 n \pi L_{F}$ (provided $L \ll L_{\text {abs }}$ ), the impact of the upstream target is very small. In this case the contributions form the both upstream target surfaces almost contract and the DTR intensity is close to the one in the absence of this target. Therefore in order to observe the increase of the DTR yield with the increase of $l$ the uncertainty in the upstream target thickness should be noticeably less than $\pi L_{F}$, which in the present case is about $5.5 \mu \mathrm{m}$.

\section{CONCLUSIONS}

In the present paper the process of the diffracted transition radiation (DTR) generated by a high-energy electron in a crystalline target in the Bragg geometry was examined. Before impinging upon the crystal the electron was assumed to penetrate through an upstream target situated on some distance $l$ from the crystal, which made the particle "half-bare." It was shown that the subsequent process of the electron's field regeneration in the region between the targets dramatically modifies the DTR angular distribution and its angle-integrated yield comparing to the case of the upstream target absence. With the increase of $l$ within the formation length $l_{F}$ (at multi$\mathrm{GeV}$ electron energies it can be macroscopically large even in the $\mathrm{x}$-ray range of frequencies) the yield grows logarithmically. Meanwhile the angular distribution narrows in such way that its maximum position shifts approximately as $l^{-1 / 2}$. First, the discussed effects were studied in the case of an inclined crystalline target when the emitted DTR was supposed to miss the upstream target on its way to the detector. Then the case of a backward radiation geometry (parallel targets), in which the emitted radiation has to penetrate through the upstream target, was investigated in more detail. 


\section{ACKNOWLEDGMENTS}

The work of S. V. T. was partiallysupported by the Project No. 0117 U004866 of the Ministry of Education and Science of Ukraine and the Projects No. L10/56-2019, No. F56/2018 and No. C-2/50-2018 of the National Academy of Sciences of Ukraine. The work of N. F. S. was partially supported by the Projects No. F56/2018 and No. C-2/50-2018 of the National Academy of Sciences of Ukraine.

[1] A. Caticha, Transition-diffracted radiation and the Čerenkov emission of x rays, Phys. Rev. A 40, 4322 (1989).

[2] X. Artru and P. Rullhusen, Parametric X-rays and diffracted transition radiation in perfect and mosaic crystals, Nucl. Instrum. Methods Phys. Res., Sect. B 145, 1 (1998); Addendum, Nucl. Instrum. Methods Phys. Res., Sect. B 173, 16 (2001).

[3] P. Rullhusen, X. Artru, and P. Dhez, Novel Radiation Sources Using Relativistic Electrons: From Infrared to $X$-rays (World Scientific, Singapore, 1998).

[4] V. G. Baryshevsky, I. D. Feranchuk, and A. P. Ulyanenkov, Parametric X-ray Radiation in Crystals. Theory, Experiments and Applications (Springer, Berlin Heidelberg, 2005).

[5] Y. N. Adischev, S. N. Arishev, A. V. Vnukov, A. V. Vukolov, A. P. Potylitsyn, S. I. Kuznetsov, V. N. Zabaev, B. N. Kalinin, V. V. Kaplin, S. R. Uglov, A. S. Kubankin, and N. N. Nasonov, Angular distribution of X-ray radiation by $500 \mathrm{MeV}$ electrons in a tungsten crystal, Nucl. Instrum. Methods Phys. Res., Sect. B 201, 114 (2003).

[6] M. L. Ter-Mikaelyan, High-Energy Electromagnetic Processes in media (Wiley, New York, 1972).

[7] G. M. Garibian and C. Yang, Quantum microscopic theory of radiation by a charged particle moving uniformly in a crystal, Sov. Phys. JETP 34, 495 (1972).

[8] V. G. Baryshevsky and I. D. Feranchuk, Transition radiation of $\gamma$ rays in a crystal, Sov. Phys. JETP 34, 502 (1972).

[9] Y. N. Adishchev, A. N. Didenko, V. V. Mun, G. A. Pleshkov, A. P. Potylitsyn, V. K. Tomchakov, S. R. Uglov, and S. A. Vorobiev, Measurements of parametric X-rays from relativistic electrons in silicon crystals, Nucl. Instrum. Methods Phys. Res., Sect. B 21, 49 (1987).

[10] A. V. Shchagin, V. I. Pristupa, and N. A. Khizhnyak, A fine structure of parametric X-ray radiation from relativistic electrons in a crystal, Phys. Lett. 148A, 485 (1990).

[11] J. Freudenberger, V. B. Gavrikov, M. Galemann, H. Genz, L. Groening, V. L. Morokhovskii, V. V. Morokhovskii, U. Nething, A. Richter, J. P. F. Sellschop, and N. F. Shul'ga, Parametric X-Ray Radiation Observed in Diamond at Low Electron Energies, Phys. Rev. Lett. 74, 2487 (1995).

[12] E. L. Feinberg, High energy successive interactions, Sov. Phys. JETP 23, 132 (1966).

[13] L. D. Landau and I. Y. Pomeranchuk, Limits of applicability of the theory of bremsstrahlung by electrons and pair creation at high energies, Dokl. Akad. Nauk SSSR 92, 535 (1953).
[14] A. B. Migdal, Bremsstrahlung and pair production in condensed media at high energies, Phys. Rev. 103, 1811 (1956).

[15] F. F. Ternovsky, On the theory of radiative processes in piecewise homogeneous media, Sov. Phys. JETP 12, 123 (1961).

[16] N. F. Shul'ga and S. P. Fomin, Suppression of radiation in an amorphous medium and in a crystal, JETP Lett. 27, 117 (1978).

[17] P. L. Anthony, R. Becker-Szendy, P. E. Bosted, M. CavalliSforza, L. P. Keller, L. A. Kelley, S. R. Klein, G. Niemi, M. L. Perl, L. S. Rochester, and J. L. White, An Accurate Measurement of the Landau-Pomeranchuk-Migdal Effect, Phys. Rev. Lett. 75, 1949 (1995).

[18] H. D. Thomsen, J. Esberg, K. Kirsebom, H. Knudsen, E. Uggerhoj, U. I. Uggerhoj, P. Sona, A. Mangiarotti, T. J. Ketel, A. Dizdar, M. M. Dalton, S. Ballestrero, and S. H. Connell, On the macroscopic formation length for $\mathrm{GeV}$ photons, Phys. Lett. B 672, 323 (2009).

[19] G. Naumenko, X. Artru, A. Potylitsyn, Y. Popov, L. Sukhikh, and M. Shevelev, "Shadowing" of the electromagnetic field of relativistic charged particles, J. Phys. Conf. Ser. 236, 012004 (2010).

[20] S. V. Trofymenko, X-ray emission by a high-energy electron with a nonequilibrium field in an ultrathin crystal, Phys. Rev. A 98, 023813 (2018).

[21] S. V. Trofymenko, Coherent X-ray radiation by an ultrarelativistic "half-bare" electron in thin crystal, IEEE Xplore Digital Library, IEEE Conference Publications, 2018 (International Conference on Mathematical Methods in Electromagnetic Theory (MMET), 2018), p. 314, DOI: 10.1109/MMET.2018.8460331.

[22] A. P. Potylitsyn and V. A. Verzilov, Parametric X-rays and transition-diffracted radiation in crystal stacks, Phys. Lett. A 209, 380 (1995).

[23] M. Y. Andreyashkin, V. N. Zabaev, V. V. Kaplin, S. R. Uglov, K. Nakayama, and I. Endo, Observation of coherent $\mathrm{X}$-ray production by $800-\mathrm{MeV}$ electrons in a periodic triple-crystal target, JETP Lett. 65, 625 (1997).

[24] S. V. Blazhevich, R. A. Zagorodniuk, and A. V. Noskov, Diffracted transition radiation of a relativistic electron in a three-layer structure, JETP 119, 641 (2014).

[25] Y. A. Goponov, S. A. Laktionova, O. O. Pligina, M. A. Sidnin, and I. E. Vnukov, Influence of real photon diffraction on parametric X-ray radiation angular distribution in thin perfect crystals, Nucl. Instrum. Methods Phys. Res., Sect. B 355, 150 (2015).

[26] I. Chaikovska, R. Chehab, X. Artru, and A. V. Shchagin, Characteristic, parametric, and diffracted transition X-ray radiation for observation of accelerated particle beam profile, Nucl. Instrum. Methods Phys. Res., Sect. B 402, 75 (2017).

[27] A. Gogolev, A. Potylitsyn, and G. Kube, A possibility of transverse beam size diagnostics using parametric X-ray radiation, J. Phys. Conf. Ser. 357, 012018 (2012).

[28] Y. Takabayashi and K. Sumitani, New method for measuring beam profiles using a parametric X-ray pinhole camera, Phys. Lett. A 377, 2577 (2013). 
[29] L. G. Sukhikh, G. Kube, and A. P. Potylitsyn, Simulation of transition radiation based beam imaging from tilted targets, Phys. Rev. Accel. Beams 20, 032802 (2017).

[30] B. Bolzon, A. Aryshev, T. Aumeyr, S. Boogert, P. Karataev, K. O. Kruchinin, T. Lefevre, S. Mazzoni, L. Nevay, M. Shevelev, N. Terunuma, J. Urakawa, and C. P. Welsch,
Very high resolution optical transition radiation imaging system: Comparison between simulation and experiment, Phys. Rev. ST Accel. Beams 18, 082803 (2015).

[31] Z. D. Pinsker, Dynamical Scattering of X-rays in Crystals (Springer, New York, 1978). 